\title{
A Variational Approach towards Solving an Ill-Posed Cauchy Problem for a Functional-Differential Equation
}

\author{
By \\ Yu. F. Dolgil and P. G. Surkov \\ (Ural Federal University and Ural Branch of the Russian Academy of Sciences, Russia)
}

\begin{abstract}
We investigate an ill-posed Cauchy problem for a linear functionaldifferential equation of retarded type on the negative half-line. Using a step-by-step procedure this problem is replaced by an inverse problem for an operator equation of the first kind in a functional space. The Tikhonov's method is then used for finding solutions. We also construct special boundary value problems for the functionaldifferential equations. Solutions of these boundary value problems determine regularized solutions of the ill-posed Cauchy problem by the step-by-step procedure.

Key Words and Phrases. Linear functional differential equation, Ill-posed problems.

2010 Mathematics Subject Classification Numbers. 34K06, 47A52.
\end{abstract}

\section{Introduction}

Let us consider the linear functional-differential equation of retarded type

$$
\frac{d x(t)}{d t}=\int_{-r}^{0}\left[d_{s} \eta(t, s)\right] x(t+s), \quad t \in(-\infty, 0],
$$

where $x:(-\infty, 0] \rightarrow \boldsymbol{R}^{n}$. We suppose that the following conditions are valid

(H1) $\eta(\cdot, s)$ is a Lebesgue locally integrable function for every fixed $s \in[-r, 0]$; in almost every fixed $t \in(-\infty, 0]$ the function $\eta(t, \cdot)$ has a bounded variation; $\operatorname{Var}_{s \in[-r, 0]} \eta(t, s), t \in(-\infty, 0]$, is a Lebesgue locally integrable function.

The Cauchy problem on the positive half-line for a functional-differential equation is well-studied. There are conditions that provide the continuous dependence of solutions on the initial functions, i.e. the correctness of the Cauchy problem [1]. The Cauchy problem on the negative half-line is ill-posed and is poorly explored. It was investigated in the work $[2,3]$ in the special case of a differential equation with one concentrated delay. The method introduced in $[2,3]$, however, can be adapted to the functional-differential equation of retarded type. 
When trying to find a solution of the Cauchy problem for system (1.1) on the negative half-line by the implicit step-by-step procedure, one needs to solve the equations

$$
U_{k+1} x_{k}=x_{k+1}, \quad k \leq-1,
$$

in a functional space. Here the linear compact operator $U_{k+1}$ acting in the space $C\left([-r, 0], \boldsymbol{R}^{n}\right)$ is given by the formula [1]

$$
\begin{aligned}
\left(U_{k+1} x_{k}\right)(\theta)= & V_{k}(\theta,-r) x_{k}(0) \\
& +\int_{-r}^{-0}\left[d_{s} \int_{0}^{r+\theta} V_{k}(\theta, \tau-r) \eta_{k}(\tau-r, s-\tau) d \tau\right] x_{k}(s), \\
& \theta \in[-r, 0] \quad(k \leq-1) .
\end{aligned}
$$

Here

$$
\begin{aligned}
& \eta_{k}(\tau, \beta)=\eta((k+1) r+\tau, \beta), \quad \beta \in[-r, 0], \\
& \eta_{k}(\tau, \beta)=\eta((k+1) r+\tau,-r), \quad \beta<-r, \\
& V_{k}(\theta, \tau)=V((k+1) r+\theta,(k+1) r+\tau), \quad \theta, \tau \in[-r, 0] \quad(k \leq-1) ;
\end{aligned}
$$

and $V(t, s), t \geq s-r$, is a matrix solution of equation (1.1) with the initial conditions

$$
\begin{aligned}
& V(t, s)=0, \quad s-r \leq t<s, \\
& V(s, s)=I_{n}, \quad s \in(-\infty, 0] .
\end{aligned}
$$

When realizing the step-by-step procedure it is necessary to find the solution $x_{k}(k \leq-1)$ of equation (1.2) for the fixed function $x_{k+1}$. This problem is illposed and for its solution we use the variational method of regularization by A. N. Tikhonov and the extension of equation (1.2) from the space $C\left([-r, 0], \boldsymbol{R}^{n}\right)$ to a separable Hilbert space.

Let us introduce the separable Hilbert space $H=L_{2}\left([-r, 0), \boldsymbol{R}^{n}\right) \times \boldsymbol{R}^{n}$ with inner product and norm

$$
(\varphi, \psi)=\psi^{\top}(0) \varphi(0)+\int_{-r}^{0} \psi^{\top}(s) \varphi(s) d s, \quad\|\varphi\|=(\varphi, \varphi)^{1 / 2} .
$$

We suppose that the following conditions are satisfied

$$
\operatorname{vrai} \sup _{t, s \in[0, r]}\left|\frac{\partial}{\partial s} \int_{0}^{t} \eta_{k}(\tau-r, s-\tau) d \tau\right|<\infty, \quad k \leq-1 .
$$


In the work [6] it is shown that using by $(\mathrm{H} 2)$ the representation of operator (1.3) can be written of the form

$$
\begin{aligned}
\left(U_{k+1} x_{k}\right)(\theta)= & V_{k}(\theta,-r) x_{k}(0) \\
& +\int_{-r}^{0} \frac{\partial}{\partial s}\left(\int_{-r}^{\theta} V_{k}(\theta, \tau) \eta_{k}(\tau, s-\tau-r) d \tau\right) x_{k}(s) d s, \\
& \theta \in[-r, 0] \quad(k \leq-1),
\end{aligned}
$$

and this operator allows a continuous extension to $H$ which is a completely continuous operator.

The variational method was used in the works of A. N. Tikhonov [4] to find regularized solutions of an integral equation of the first kind in the space of squared integrable functions $L_{2}\left([-r, 0], \boldsymbol{R}^{n}\right)$. It was shown that the regularized solution of the integral equation was a solution of the special boundary value problem for an integro-differential equation. A. N. Tikhonov used the smooth stabilizing functionals which generate a metrization of the sets $W_{2}^{m}\left([-r, 0], \boldsymbol{R}^{n}\right)$ that majorizes the metric of the space $L_{2}\left([-r, 0], \boldsymbol{R}^{n}\right)[4,5]$.

The operator $U_{k+1}(k \leq-1)$ of equation (1.2) is not integral. By taking into account the representation of this operator, we change the space $L_{2}\left([-r, 0], \boldsymbol{R}^{n}\right)$ on the space $H$ and choose the stabilizing functional of the form

$$
\begin{aligned}
\Omega[x]= & \sum_{j=0}^{m-1} x^{(j) \top}(0) Q_{j} x^{(j)}(0)+\int_{-r}^{0} \sum_{j=0}^{m} x^{(j) \top}(s) P_{j}(s) x^{(j)}(s) d s, \\
& x \in W_{2}^{m}\left([-r, 0], \boldsymbol{R}^{n}\right), m \geq 1,
\end{aligned}
$$

where the notations $x^{(0)}(s)=x(s), x^{(j)}(s)=d^{j} x(s) / d s^{j}, s \in[-r, 0], j \geq 1$, are used. We suppose that the following conditions are satisfied

(H3) $Q_{j}, 0 \leq j \leq m-1$, and $P_{l}(\theta), \theta \in[-r, 0], 0 \leq l \leq m$, are symmetric positive defined matrices, columns of the matrix-functions $P_{0}$ are elements of $C\left([-r, 0], \boldsymbol{R}^{n}\right)$, columns of the matrix-functions $P_{l}$ are elements of the space $W_{2}^{l}\left([-r, 0], \boldsymbol{R}^{n}\right), 1 \leq l \leq m$.

In the variational method of solving the equation (1.2) for fixed positive values of the regularization parameter $\alpha$ and for any function $x_{k+1} \in H$, we define the function $x_{k}=x_{k \alpha} \in W_{2}^{m}\left([-r, 0], \boldsymbol{R}^{n}\right)$ minimizing the functional

$$
M^{\alpha}\left[x_{k+1}, x_{k}\right]=\left(U_{k+1} x_{k}-x_{k+1}, U_{k+1} x_{k}-x_{k+1}\right)+\alpha \Omega\left[x_{k}\right] \quad(k \leq-1) .
$$

If there exists the function $x_{k \alpha}$ minimizing the smoothing functional then it is referred to as a regularized solution of equation (1.2). The value of the regularizing operator of equation (1.2) for the arbitrary function $x_{k+1} \in H$ is defined by the formula

$$
R_{k+1}\left(x_{k+1}, \alpha\right)=x_{k \alpha} \quad(k \leq-1) .
$$


We define the solution of the ill-posed Cauchy problem for system (1.1) with the initial function $\varphi \in H$ on the negative half-line by the implicit step-bystep procedure

$$
x_{k}=R_{k+1}\left(x_{k+1}, \alpha\right), \quad k \leq-1, \quad x_{0}=\varphi .
$$

This procedure defines the sequence $x_{k}(\cdot, \varphi, \alpha), k \leq-1$, which can be used to find the regularized solutions of system (1.1) on the negative half-line by the formulas $x(k r+\theta, \varphi, \alpha)=x_{k}(\theta, \varphi, \alpha), \theta \in[-r, 0), k \leq-1, x(\theta, \varphi, \alpha)=\varphi(\theta)$, $\theta \in[-r, 0]$.

In the present paper it is shown that the regularized solution $x_{k \alpha}(k \leq-1)$ of equation (1.2) is a component of a solution of a special boundary value problem for the system of functional-differential equations.

Main Theorem. Let the conditions (H1)-(H3) be satisfied and let Ker $U_{k+1}=\{0\} \quad(k \leq-1)$. Then the solution of the boundary value problem for the following system of functional-differential equations

$$
\begin{aligned}
& \alpha \sum_{j=0}^{m}(-1)^{j} \frac{d^{j}}{d \theta^{j}}\left(P_{j}(\theta) x_{k}^{(j)}(\theta)\right) \\
& \quad+\frac{d}{d \theta} \int_{-r}^{\theta}\left(\eta_{k}(s, \theta-s-r)-\eta_{k}(s,-r)\right)^{\top}\left(\hat{\chi}_{k}(s)-\hat{z}_{k}(s)\right) d s=0, \\
& \frac{d \hat{\chi}_{k}(\theta)}{d \theta}=\int_{\theta}^{0}\left[\left.d_{s} \eta_{k}^{\top}(\tau, \theta-s)\right|_{\tau=s}\right] \hat{\chi}_{k}(s)-\chi_{k}(\theta), \\
& \frac{d \hat{z}_{k}(\theta)}{d \theta}=\int_{\theta}^{0}\left[\left.d_{s} \eta_{k}^{\top}(\tau, \theta-s)\right|_{\tau=s}\right] \hat{z}_{k}(s)-x_{k+1}(\theta), \\
& \frac{d \chi_{k}(\theta)}{d \theta}=\int_{-r-\theta}^{0}\left[d_{s} \eta_{k}(\theta, s)\right] \chi_{k}(\theta+s)+\int_{\theta}^{0}\left[d_{s} \eta_{k}(\theta, s-\theta-r)\right] x_{k}(s),
\end{aligned}
$$

with the boundary conditions

$$
\begin{aligned}
& \alpha\left(Q_{0} x_{k}(0)+\left.\sum_{j=1}^{m}(-1)^{j-1} \frac{d^{j-1}}{d \theta^{j-1}}\left(P_{j}(\theta) x_{k}^{(j)}(\theta)\right)\right|_{\theta=0}\right) \\
& \quad+\hat{\chi}_{k}(-r)-\hat{z}_{k}(-r)=0, \\
& Q_{l} x_{k}^{(l)}(0)+\left.\sum_{j=l+1}^{m}(-1)^{j-l-1} \frac{d^{j-l-1}}{d \theta^{j-l-1}}\left(P_{j}(\theta) x_{k}^{(j)}(\theta)\right)\right|_{\theta=0}=0, \\
& 1 \leq l \leq m-1, m \geq 2,
\end{aligned}
$$




$$
\begin{aligned}
& \left.\sum_{j=l+1}^{m}(-1)^{j-l-1} \frac{d^{j-l-1}}{d \theta^{j-l-1}}\left(P_{j}(\theta) x_{k}^{(j)}(\theta)\right)\right|_{\theta=-r}=0, \quad 0 \leq l \leq m-1, \\
& \hat{\chi}_{k}(0)=\chi_{k}(0), \quad \hat{z}_{k}(0)=x_{k+1}(0), \quad \chi_{k}(-r)=x_{k}(0), \quad(k \leq-1),
\end{aligned}
$$

exists and is unique. When $m=1$, condition (1.11) is absent. The component $x_{k}=x_{k \alpha} \quad(k \leq-1)$ coincides with the solution of the variation problem of minimization of the smoothing functional $M^{\alpha}$.

The component $\chi_{k \alpha}$ of the solution of the boundary value problem satisfies the condition $\chi_{k \alpha}=U_{k+1} x_{k \alpha}(k \leq-1)$. Here $\alpha$ is the regularization parameter and $x_{0}=\varphi \in H$ is a given function.

\section{A boundary value problem for an integro-differential equation}

Without loss of generality, we solve the minimization problem of the smoothing functional for $k=-1$. Let us introduce new notations $x_{-1}=x$, $x_{0}=\varphi, U_{0}=U$. Then the smoothing functional takes the form

$$
M^{\alpha}[\varphi, x]=(U x-\varphi, U x-\varphi)+\alpha \Omega[x], \quad x \in W_{2}^{m}\left([-r, 0], \boldsymbol{R}^{n}\right),
$$

and the operator $U$ is given by the formula

$$
\begin{gathered}
(U x)(\theta)=V(\theta,-r) x(0)+\int_{-r}^{0} \frac{\partial}{\partial s}\left(\int_{-r}^{\theta} V(\theta, \tau) \eta(\tau-r, s-\tau-r) d \tau\right) x(s) d s, \\
\theta \in[-r, 0], \varphi \in H .
\end{gathered}
$$

Lemma 1. Let the conditions $(\mathrm{H} 1)-(\mathrm{H} 3)$ hold. Then the function minimizing the smoothing functional (2.1) exists if and only if there exists a solution of the integro-differential equation

$$
U^{*}(U x-\varphi)(\theta)+\alpha \sum_{j=0}^{m}(-1)^{j} \frac{d^{j}}{d \theta^{j}}\left(P_{j}(\theta) x^{(j)}(\theta)\right)=0, \quad \theta \in[-r, 0),
$$

that can be extended by continuity with its derivatives $x^{(j)}, 1 \leq j \leq 2 m-1$, to the interval $[-r, 0]$ and satisfies the boundary conditions

$$
\begin{aligned}
& U^{*}(U x-\varphi)(0) \\
& \quad+\alpha\left(Q_{0} x(0)+\left.\sum_{j=1}^{m}(-1)^{j-1} \frac{d^{j-1}}{d \theta^{j-1}}\left(P_{j}(\theta) x^{(j)}(\theta)\right)\right|_{\theta=0}\right)=0, \quad \varphi \in H, \\
& Q_{l} x^{(l)}(0)+\left.\sum_{j=l+1}^{m}(-1)^{j-l-1} \frac{d^{j-l-1}}{d \theta^{j-l-1}}\left(P_{j}(\theta) x^{(j)}(\theta)\right)\right|_{\theta=0}=0, \\
& 1 \leq l \leq m-1, m \geq 2
\end{aligned}
$$




$$
\left.\sum_{j=l+1}^{m}(-1)^{j-l-1} \frac{d^{j-l-1}}{d \theta^{j-l-1}}\left(P_{j}(\theta) x^{(j)}(\theta)\right)\right|_{\theta=-r}=0, \quad 0 \leq l \leq m-1 .
$$

When $m=1$, the second boundary condition is absent. If there exists the solution $x$ of the boundary value problem (2.2), (2.3), then it belongs to $W_{2}^{2 m}\left([-r, 0], \boldsymbol{R}^{n}\right)$ and coincides with the function $x_{\alpha}$ minimizing the smoothing functional (2.1).

Proof. By using the equality

$$
(U x, y)=\left(x, U^{*} y\right), \quad x, y \in H,
$$

we find a representation of the adjoint operator $U^{*}: H \rightarrow H$. We have

$$
\begin{aligned}
\left(U^{*} y\right)(0)= & V^{\top}(0,-r) y(0)+\int_{-r}^{0} V^{\top}(s,-r,) y(s) d s, \\
\left(U^{*} y\right)(\theta)= & \frac{d}{d \theta}\left(\int_{-r}^{0} \eta^{\top}(\tau, \theta-\tau-r) V^{\top}(0, \tau) d \tau\right) y(0) \\
& +\int_{-r}^{0} \frac{d}{d \theta}\left(\int_{-r}^{s} \eta^{\top}(\tau, \theta-\tau-r) V^{\top}(s, \tau) d \tau\right) y(s) d s, \quad \theta \in[-r, 0) .
\end{aligned}
$$

From the representations of the operators $U$ and $U^{*}$, it follows that equation (2.2) is an integro-differential equation.

The function $x \in W_{2}^{m}\left([-r, 0], \boldsymbol{R}^{n}\right)$ minimizes the smoothing functional if and only if the inequality

$$
\begin{aligned}
M^{\alpha}(x+\tilde{x}, \varphi)= & M^{\alpha}(x, \varphi)+2\left((U x-\varphi, U \tilde{x})+\alpha \sum_{j=0}^{m-1} \tilde{x}^{(j) \top}(0) Q_{j} x^{(j)}(0)\right. \\
& \left.+\alpha \int_{-r}^{0} \sum_{j=0}^{m} \tilde{x}^{(j) \top}(s) P_{j}(s) x^{(j)}(s) d s\right)+(U \tilde{x}, U \tilde{x})+\alpha \Omega[\tilde{x}] \\
\geq & M^{\alpha}(x, \varphi)
\end{aligned}
$$

holds for any function $\tilde{x} \in W_{2}^{m}\left([-r, 0], \boldsymbol{R}^{n}\right)$. The necessary and sufficient condition for this is the fulfillment of equality

$$
\begin{array}{r}
\left(U^{*}(U x-\varphi), \tilde{x}\right)+\alpha \sum_{j=0}^{m-1} \tilde{x}^{(j) \top}(0) Q_{j} x^{(j)}(0) \\
\quad+\alpha \int_{-r}^{0} \sum_{j=0}^{m} \tilde{x}^{(j) \top}(s) P_{j}(s) x^{(j)}(s) d s=0
\end{array}
$$

for any function $\tilde{x} \in W_{2}^{m}\left([-r, 0], \boldsymbol{R}^{n}\right)$. 
By using the integration by parts formula, we obtain

$$
\begin{aligned}
\int_{-r}^{0} \tilde{x}^{(j) \top}(\theta) P_{j}(\theta) x^{(j)}(\theta) d \theta \\
\quad=\int_{-r}^{0} \tilde{x}^{(j) \top}(\theta) \frac{d}{d \theta}\left(J P_{j}(\cdot) x^{(j)}(\cdot)\right)(\theta) d \theta \\
=\tilde{x}^{(j) \top}(0)\left(J P_{j}(\cdot) x^{(j)}(\cdot)\right)(0)-\int_{-r}^{0} \tilde{x}^{(j+1) \top}(\theta)\left(J P_{j}(\cdot) x^{(j)}(\cdot)\right)(\theta) d \theta \\
=\sum_{k=j}^{m-1}(-1)^{k-j} \tilde{x}^{(k) \top}(0)\left(J^{k+1-j} P_{j}(\cdot) x^{(j)}(\cdot)\right)(0) \\
\quad+(-1)^{m-j} \int_{-r}^{0} \tilde{x}^{(m) \top}(\theta)\left(J^{m-j} P_{j}(\cdot) x^{(j)}(\cdot)\right)(\theta) d \theta, \quad 0 \leq j \leq m-1 .
\end{aligned}
$$

Here the integral operator $J$ is defined by the formula

$$
(J x)(\theta)=\int_{-r}^{\theta} x(s) d s, \quad \theta \in[-r, 0], x \in H .
$$

By using the formulas (2.5), we have

$$
\begin{aligned}
& \int_{-r}^{0} \sum_{j=0}^{m} \tilde{x}^{(j) \top}(\theta) P_{j}(\theta) x^{(j)}(\theta) d \theta \\
& =\sum_{k=0}^{m-1} \tilde{x}^{(k) \top}(0) \sum_{j=0}^{k}(-1)^{k-j} \tilde{x}^{(k) \top}(0)\left(J^{k+1-j} P_{j}(\cdot) x^{(j)}(\cdot)\right)(0) \\
& \quad+\int_{-r}^{0} \tilde{x}^{(m) \top}(\theta) \sum_{j=0}^{m}(-1)^{m-j}\left(J^{m-j} P_{j}(\cdot) x^{(j)}(\cdot)\right)(\theta) d \theta
\end{aligned}
$$

Here $J^{0}=I$ is the identity operator.

Let us introduce the function $\psi(\theta)=\left(U^{*}(U x-\varphi)\right)(\theta), \theta \in[-r, 0], \psi \in H$, and simplify the formula of the inner product in equality (2.4)

$$
\begin{aligned}
(\psi, \tilde{x}) & =\tilde{x}^{\top}(0) \psi(0)+\int_{-r}^{0} \tilde{x}^{\top}(\theta) \psi(\theta) d \theta \\
& =\sum_{k=0}^{m-1}(-1)^{k} \tilde{x}^{(k) \top}(0)\left(J^{k+1} \psi\right)(0)+(-1)^{m} \int_{-r}^{0} \tilde{x}^{(m) \top}(\theta)\left(J^{m} \psi\right)(\theta) d \theta .
\end{aligned}
$$


As a result equality (2.4) takes the form

$$
\begin{aligned}
& \sum_{k=0}^{m-1} \tilde{x}^{(k) \top}(0)\left((-1)^{k}\left(J^{k+1} \psi\right)(0)+\alpha Q_{k} \tilde{x}^{(k)}(0)\right. \\
& \left.\quad+\alpha \sum_{j=0}^{k}(-1)^{k-j}\left(J^{k+1-j} P_{j}(\cdot) x^{(j)}(\cdot)\right)(0)\right) \\
& +\int_{-r}^{0} \tilde{x}^{(m) \top}(\theta)\left(\alpha \sum_{j=0}^{m}(-1)^{m-j}\left(J^{m-j} P_{j}(\cdot) x^{(j)}(\cdot)\right)(\theta)+(-1)^{m}\left(J^{m} \psi\right)(\theta)\right) d \theta \\
& +\tilde{x}^{\top}(0) \psi(0)=0 .
\end{aligned}
$$

Since $\tilde{x} \in W_{2}^{m}\left([-r, 0], \boldsymbol{R}^{n}\right)$ is arbitrary and because of the definition of the function $\psi$ we obtain

$$
\begin{aligned}
& (-1)^{m}\left(J^{m} U^{*}(U x-\varphi)\right)(\theta)+\alpha \sum_{j=0}^{m}(-1)^{m-j}\left(J^{m-j} P_{j}(\cdot) x^{(j)}(\cdot)\right)(\theta)=0 \\
& \theta \in[-r, 0) \\
& (-1)^{k}\left(J^{k+1} U^{*}(U x-\varphi)\right)(0)+\alpha Q_{k} x^{(k)}(0) \\
& \quad+\alpha \sum_{j=0}^{k}(-1)^{k-j}\left(J^{k+1-j} P_{j}(\cdot) x^{(j)}(\cdot)\right)(0)=0, \quad 1 \leq k \leq m-1 \\
& \left(U^{*}(U x-\varphi)\right)(0)+\left(J U^{*}(U x-\varphi)\right)(0)+\alpha Q_{0} x(0)+\alpha\left(J P_{0}(\cdot) x(\cdot)\right)(0)=0
\end{aligned}
$$

from the last equality. When $m=1$, equality (2.7) is absent. From (2.6)-(2.8) it follows that the function $x$ minimizing the smoothing functional belongs to the space $W_{2}^{2 m}\left([-r, 0], \boldsymbol{R}^{n}\right)$ and satisfies integro-differential equation (2.6) with the boundary conditions (2.7), (2.8).

If we differentiate $m$ times equality (2.6), we obtain the integro-differential equation

$$
(-1)^{m}\left(U^{*}(U x-\varphi)\right)(\theta)+\alpha \sum_{j=0}^{m}(-1)^{m-j}\left(J^{-j} P_{j}(\cdot) x^{(j)}(\cdot)\right)(\theta)=0, \quad \theta \in[-r, 0),
$$

with the boundary conditions

$$
\sum_{j=m-k}^{m}(-1)^{m-j}\left(J^{m-j-k} P_{j}(\cdot) x^{(j)}(\cdot)\right)(-r)=0, \quad 0 \leq k \leq m-1 .
$$


Here $J^{-j}$ is the operator of differentiation

$$
\left(J^{-j} y\right)(\theta)=\frac{d^{j} y(\theta)}{d \theta^{j}}, \quad \theta \in[-r, 0], 1 \leq j \leq m, y \in W_{2}^{m}\left([-r, 0], \boldsymbol{R}^{n}\right) .
$$

By taking into account the definition of the function $\psi$ we conclude that the integro-differential equation (2.6) is equivalent to the following boundary value problem of integro-differential equations

$$
\begin{aligned}
U^{*}(U x-\varphi)(\theta)+\alpha \sum_{j=0}^{m}(-1)^{j} \frac{d^{j}}{d \theta^{j}}\left(P_{j}(\theta) x^{(j)}(\theta)\right)=0, & \theta \in[-r, 0), \\
\left.\sum_{j=l+1}^{m}(-1)^{j-l-1} \frac{d^{j-l-1}}{d \theta^{j-l-1}}\left(P_{j}(\theta) x^{(j)}(\theta)\right)\right|_{\theta=-r}=0, & 0 \leq l \leq m-1 .
\end{aligned}
$$

If we differentiate $q$ times equality (2.6) and suppose that $\theta=0$, we obtain

$$
(-1)^{m}\left(J^{m-j} \psi\right)(0)+\alpha \sum_{l=0}^{m}(-1)^{m-l}\left(J^{m-l-j} P_{l}(\cdot) x^{(l)}(\cdot)\right)(0)=0, \quad 0 \leq j \leq m-1 .
$$

By taking into account the last equalities we bring the boundary conditions (2.7) and (2.8) to the form in the statement of Lemma 1.

\section{Existence and uniqueness of the boundary value problem for the integro-differential equation}

Let us introduce the unbounded operator $L: H \rightarrow H$ defined by the formulas

$$
\begin{aligned}
& (L x)(\theta)=\sum_{j=0}^{m}(-1)^{j} \frac{d^{j}}{d \theta^{j}}\left(P_{j}(\theta) x^{(j)}(\theta)\right), \quad \theta \in[-r, 0) \\
& (L x)(0)=Q_{0} x(0)+\left.\sum_{j=1}^{m}(-1)^{j-1} \frac{d^{j-1}}{d \theta^{j-1}}\left(P_{j}(\theta) x^{(j)}(\theta)\right)\right|_{\theta=0}
\end{aligned}
$$

with its domain

$$
\begin{gathered}
\mathscr{D}(L)=\left\{x: x \in W_{2}^{2 m}\left([-r, 0], \boldsymbol{R}^{n}\right), F_{l}^{1}(x)=0,1 \leq l \leq m-1, m \geq 2,\right. \\
\left.F_{l}^{2}(x)=0,0 \leq l \leq m-1\right\},
\end{gathered}
$$


which is dense in the space $H$. Here

$$
\begin{aligned}
F_{l}^{1}(x)= & Q_{l} x^{(l)}(0)+\left.\sum_{j=l+1}^{m}(-1)^{j-l-1} \frac{d^{j-l-1}}{d \theta^{j-l-1}}\left(P_{j}(\theta) x^{(j)}(\theta)\right)\right|_{\theta=0}, \\
& 1 \leq l \leq m-1, m \geq 2, \\
F_{l}^{2}(x)= & \left.\sum_{j=l+1}^{m}(-1)^{j-l-1} \frac{d^{j-l-1}}{d \theta^{j-l-1}}\left(P_{j}(\theta) x^{(j)}(\theta)\right)\right|_{\theta=-r}, \quad 0 \leq l \leq m-1 .
\end{aligned}
$$

When $m=1$, we have $\mathscr{D}(L)=\left\{x: x \in W_{2}^{2}\left([-r, 0], \boldsymbol{R}^{n}\right), F_{0}^{2}(x)=0\right\}$.

By using the definition of the operator $L$, the boundary value problem for integro-differential equations can be written in operator form as follows

$$
U^{*}(U x-\varphi)(\theta)+\alpha(L x)(\theta)=0, \quad \theta \in[-r, 0] .
$$

Lemma 2. Let conditions (H1)-(H3) hold. Then the unbounded operator $L: \mathscr{D}(L) \rightarrow H$ is self-adjoint and positive.

Proof. Let us show that the equality

$$
(L x, y)=(x, L y), \quad x, y \in \mathscr{D}(L)
$$

holds, by using the definition of the adjoint operator [8]. By taking into account the definitions of the inner product and the operator $L$, we obtain

$$
\begin{aligned}
(L x, y)= & y^{\top}(0)\left(Q_{0} x(0)+\left.\sum_{j=1}^{m}(-1)^{j-1} \frac{d^{j-1}}{d \theta^{j-1}}\left(P_{j}(\theta) x^{(j)}(\theta)\right)\right|_{\theta=0}\right) \\
& +\int_{-r}^{0} y^{\top}(\theta) \sum_{j=0}^{m}(-1)^{j} \frac{d^{j}}{d \theta^{j}}\left(P_{j}(\theta) x^{(j)}(\theta)\right) d \theta .
\end{aligned}
$$

By using the integration by parts formula, we have

$$
\begin{aligned}
\int_{-r}^{0} y^{\top}(\theta) \frac{d^{j}}{d \theta^{j}}\left(P_{j}(\theta) x^{(j)}(\theta)\right) d \theta & \\
= & \left.\sum_{l=1}^{j}(-1)^{l-1} y^{(l-1) \top}(\theta) \frac{d^{j-l}}{d \theta^{j-l}}\left(P_{j}(\theta) x^{(j)}(\theta)\right)\right|_{-r} ^{0} \\
& +(-1)^{j} \int_{-r}^{0} y^{(j) \top}(\theta) P_{j}(\theta) x^{(j)}(\theta) d \theta, \quad 1 \leq j \leq m .
\end{aligned}
$$


This equality gives us

$$
\begin{aligned}
\int_{-r}^{0} y^{\top} & (\theta) \sum_{j=0}^{m}(-1)^{j} \frac{d^{j}}{d \theta^{j}}\left(P_{j}(\theta) x^{(j)}(\theta)\right) d \theta \\
= & \left.\sum_{j=1}^{m} \sum_{l=1}^{j}(-1)^{j+l-1} y^{(l-1) \top}(\theta) \frac{d^{j-l}}{d \theta^{j-l}}\left(P_{j}(\theta) x^{(j)}(\theta)\right)\right|_{-r} ^{0} \\
& +\int_{-r}^{0} y^{\top}(\theta) P_{0}(\theta) x(\theta) d \theta+\sum_{j=1}^{m} \int_{-r}^{0}\left(y^{(j)}\right)^{\top}(\theta) P_{j}(\theta) x^{(j)}(\theta) d \theta .
\end{aligned}
$$

When $m \geq 2$, we reduce the following expression by changing the order of summation

$$
\begin{aligned}
\left.\sum_{j=1}^{m} \sum_{l=1}^{j}(-1)^{j+l-1} y^{(l-1) \top}(\theta) \frac{d^{j-l}}{d \theta^{j-l}}\left(P_{j}(\theta) x^{(j)}(\theta)\right)\right|_{-r} ^{0} \\
\quad=-\left.\sum_{l=0}^{m-1} y^{(l) \top}(\theta) \sum_{j=l+1}^{m}(-1)^{j-l-1} \frac{d^{j-l-1}}{d \theta^{j-l-1}}\left(P_{j}(\theta) x^{j}(\theta)\right)\right|_{-r} ^{0} \\
=-\left.\sum_{l=0}^{m-1} y^{(l) \top}(\theta) \sum_{j=l+1}^{m}(-1)^{j-l-1} \frac{d^{j-l-1}}{d \theta^{j-l-1}}\left(P_{j}(\theta) x^{j}(\theta)\right)\right|_{\theta=0} \\
=-\left.y^{\top}(0) \sum_{j=1}^{m}(-1)^{j-1} \frac{d^{j-1}}{d \theta^{j-1}}\left(P_{j}(\theta) x^{j}(\theta)\right)\right|_{\theta=0}+\sum_{j=1}^{m-1} y^{(j) \top}(0) Q_{j} x^{(j)}(0) .
\end{aligned}
$$

As a result, we find

$$
(L x, y)=\sum_{j=0}^{m-1} y^{(j) \top}(0) Q_{j} x^{(j)}(0)+\sum_{j=0}^{m} \int_{-r}^{0} y^{(j) \top}(\theta) P_{j}(\theta) x^{(j)}(\theta) d \theta .
$$

By using the integration by parts formula, we have

$$
\begin{aligned}
\int_{-r}^{0} y^{(j) \top}(\theta) P_{j}(\theta) x^{(j)}(\theta) d \theta & \\
= & \left.\sum_{l=1}^{j}(-1)^{l-1} \frac{d^{l-1}}{d \theta^{l-1}}\left(P_{j}(\theta) y^{(j)}(\theta)\right)^{\top} x^{(j-l)}(\theta)\right|_{-r} ^{0} \\
& +(-1)^{j} \int_{-r}^{0} \frac{d^{j}}{d \theta^{j}}\left(P_{j}(\theta) y^{(j)}(\theta)\right)^{\top} x(\theta) d \theta, \quad 1 \leq j \leq m .
\end{aligned}
$$


The latest equality and the equation (3.2) give us

$$
\begin{aligned}
(L x, y)= & \sum_{j=0}^{m-1} y^{(j) \top}(0) Q_{j} x^{(j)}(0) \\
& +\left.\sum_{j=1}^{m} \sum_{l=1}^{j}(-1)^{l-1} \frac{d^{l-1}}{d \theta^{l-1}}\left(P_{j}(\theta) y^{(j)}(\theta)\right)^{\top} x^{(j-l)}(\theta)\right|_{-r} ^{0} \\
& +\sum_{j=0}^{m}(-1)^{j} \int_{-r}^{0} \frac{d^{j}}{d \theta^{j}}\left(P_{j}(\theta) y^{(j)}(\theta)\right)^{\top} x(\theta) d \theta .
\end{aligned}
$$

We reduce the expression

$$
\begin{aligned}
\sum_{j=1}^{m} & \left.\sum_{l=1}^{j}(-1)^{l-1} \frac{d^{l-1}}{d \theta^{l-1}}\left(P_{j}(\theta) y^{(j) \top}(\theta) x^{(j-l)}(\theta)\right)\right|_{-r} ^{0} \\
& =\left.\sum_{j=1}^{m} \sum_{l=0}^{j-1}(-1)^{j-l-1} \frac{d^{j-l-1}}{d \theta^{j-l-1}}\left(P_{j}(\theta) y^{(j)}(\theta)\right)^{\top} x^{(l)}(\theta)\right|_{-r} ^{0} \\
& =\left.\sum_{l=0}^{m-1} \sum_{j=l+1}^{m}(-1)^{j-l-1} \frac{d^{j-l-1}}{d \theta^{j-l-1}}\left(P_{j}(\theta) y^{(j)}(\theta)\right)^{\top} x^{(l)}(\theta)\right|_{-r} ^{0} \\
& =\left.\sum_{j=1}^{m}(-1)^{j-1} \frac{d^{j-1}}{d \theta^{j-1}}\left(P_{j}(\theta) y^{(j)}(\theta)\right)^{\top} x(\theta)\right|_{\theta=0}-\sum_{j=1}^{m-1} y^{(j) \top}(0) Q_{j} x^{(j)}(0) .
\end{aligned}
$$

As a result, we obtain the equality

$$
\begin{aligned}
(L x, y)= & \left(y^{\top}(0) Q_{0}+\left.\sum_{j=1}^{m}(-1)^{j-1} \frac{d^{j-1}}{d \theta^{j-1}}\left(P_{j}(\theta) y^{(j)}(\theta)\right)^{\top}\right|_{\theta=0}\right) x(0) \\
& +\int_{-r}^{0} \sum_{j=0}^{m}(-1)^{j} \frac{d^{j}}{d \theta^{j}}\left(P_{j}(\theta) y^{(j)}(\theta)\right)^{\top} x(\theta) d \theta=(x, L y), \quad x, y \in \mathscr{D}(L)
\end{aligned}
$$

Thus, the operator $L$ is self-adjoint.

The equality (3.2) and a positive definiteness of the matrices $Q_{j}$, $j=0, \ldots, m-1$, and $P_{j}(\theta), \theta \in[-r, 0], j=0, \ldots, m$, lead to

$$
(L x, x)=\sum_{j=0}^{m-1} x^{(j) \top}(0) Q_{j} x^{(j)}(0)+\sum_{j=0}^{m} \int_{-r}^{0} x^{(j) \top}(\theta) P_{j}(\theta) x^{(j)}(\theta) d \theta>0,
$$

for any $x \in \mathscr{D}(L), x \neq 0$. Consequently, the operator $L$ is positive. 
Lemma 3. Let conditions $(\mathrm{H} 1)-(\mathrm{H} 3)$ be satisfied. Then the operator $L^{-1}: H \rightarrow H$ is bounded and determined by the formula

$$
\left(L^{-1} y\right)(\theta)=G(\theta, 0) y(0)+\int_{-r}^{0} G(\theta, s) y(s) d s, \quad \theta \in[-r, 0],
$$

where

(i) $G(\theta, s)=G^{\top}(s, \theta), \theta, s \in[-r, 0]$;

(ii) for a fixed $s \in[-r, 0]$, the matrix-function $G(\theta, s), \theta \in[-r, 0)$, has almost everywhere the $2 m$-th partial derivative which is square integrable on $[-r, 0)$;

(iii) for a fixed $s \in[-r, 0)$, the discontinuity of the partial derivative $G_{\theta}^{(2 m-1)}(\theta, s), \theta \in[-r, 0)$, in the point $\theta=s$ is determined by the equality

$$
\left.\frac{\partial^{m-1}}{\partial \theta^{m-1}}\left(P_{m}(\theta) G_{\theta}^{(m)}(\theta, s)\right)\right|_{\theta=s+0}-\left.\frac{\partial^{m-1}}{\partial \theta^{m-1}}\left(P_{m}(\theta) G_{\theta}^{(m)}(\theta, s)\right)\right|_{\theta=s-0}=(-1)^{m} I_{n}
$$

here $G_{\theta}^{(m)}(\theta, s)=\partial^{m} G(\theta, s) / \partial \theta^{m}$;

(iv) for a fixed $s \in[-r, 0]$, the matrix-function $G(\theta, s), \theta \in[-r, 0)$, satisfies almost everywhere the matrix ordinary differential equation

$$
\sum_{j=0}^{m} \frac{d^{j}}{d \theta^{j}}\left(P_{j}(\theta) G_{\theta}^{(j)}(\theta, s)\right)=0
$$

as well as the boundary conditions

$$
\begin{gathered}
\left.Q_{k} \frac{\partial^{k} G(\theta, s)}{\partial \theta^{k}}\right|_{\theta=-0}+\left.\sum_{j=k+1}^{m}(-1)^{j-k-1} \frac{\partial^{j-k-1}}{\partial \theta^{j-k-1}}\left(P_{j}(\theta) G_{\theta}^{(j)}(\theta, s)\right)\right|_{\theta=-0}=0, \\
1 \leq k \leq m-1, m \geq 2, \\
\left.\sum_{j=k+1}^{m}(-1)^{j-k-1} \frac{\partial^{j-k-1}}{\partial \theta^{j-k-1}}\left(P_{j}(\theta) G_{\theta}^{(j)}(\theta, s)\right)\right|_{\theta=-r}=0, \\
0 \leq k \leq m-1, s \in[-r, 0),
\end{gathered}
$$

when $m=1$, equality (3.6) is absent;

(v) the matrix-function $G(\theta, 0), \theta \in[-r, 0)$, is defined by the formula

$$
G(\theta, 0)=-\left.\sum_{l=1}^{m}(-1)^{l-1} \frac{\partial^{l-1}}{\partial s^{l-1}}\left(G_{s}^{(l)}(\theta, s) P_{l}(s)\right)\right|_{s=-0} Q_{0}^{-1}
$$


and the matrix $G(0,0)$ is defined by the formula

$$
G(0,0)=Q_{0}^{-1}\left(I_{n}-\left.\sum_{l=1}^{m}(-1)^{l-1} \frac{\partial^{l-1}}{\partial \theta^{l-1}}\left(P_{l}(\theta) G_{\theta}^{(l)}(\theta, 0)\right)\right|_{\theta=-0}\right) .
$$

Proof. Here we use the methods from the work [7]. It is follows from Lemma 2 that the equation $(L x)(\theta)=y(\theta), \theta \in[-r, 0]$, has a unique solution for any function $y \in H$. The representation of this solution $x(\theta)=\left(L^{-1} y\right)(\theta)$, $\theta \in[-r, 0]$, takes the form

$$
\left(L^{-1} y\right)(\theta)=G_{0}(\theta) y(0)+\int_{-r}^{0} G(\theta, s) y(s) d s, \quad \theta \in[-r, 0] .
$$

The requirement of self-adjointness of the operator $L^{-1}$ is given by the equality $\left(L^{-1} y, z\right)=\left(y, L^{-1} z\right), y, z \in H$, from which the expressions for the coefficients of representation of the operator $L^{-1}$ follow:

$$
\begin{aligned}
& G^{\top}(s, \theta)=G(\theta, s), \quad \theta, s \in[-r, 0), \quad G_{0}(\theta)=G^{\top}(0, \theta), \quad \theta \in[-r, 0), \\
& G_{0}^{\top}(s)=G(0, s), \quad s \in[-r, 0), \quad G_{0}^{\top}(0)=G_{0}(0) .
\end{aligned}
$$

Therefore one can conclude that $G_{0}(\theta)=G(\theta, 0), \theta \in[-r, 0]$, where $G(\theta, s)=$ $G^{\top}(s, \theta), \theta, s \in[-r, 0]$. As a result, we obtain formula (3.3) of the operator $L^{-1}$.

The existence of $2 m$-th partial derivative $G_{\theta}^{(2 m)}(\theta, s), \theta \in[-r, 0), s \in[-r, 0]$, which is square integrable on $[-r, 0)$, follows from the definition $\mathscr{D}(L)$.

For finding the function $G$ we use the equality

$$
L \circ L^{-1} y=y, \quad y \in H .
$$

This holds when $L^{-1} y \in \mathscr{D}(L)$ for $y \in H$. By taking into account the definition of the set $\mathscr{D}(L)$, we derive the conditions

$$
\begin{aligned}
& Q_{k}\left(\left.\frac{\partial^{k} G(\theta, 0)}{\partial \theta^{k}}\right|_{\theta=-0} y(0)+\left.\int_{-r}^{0} \frac{\partial^{k} G(\theta, s)}{\partial \theta^{k}}\right|_{\theta=-0} y(s) d s\right) \\
& \quad+\left.\sum_{j=k+1}^{m}(-1)^{j-k-1} \frac{\partial^{j-k-1}}{\partial \theta^{j-k-1}}\left(P_{j}(\theta) G_{\theta}^{(j)}(\theta, 0)\right)\right|_{\theta=-0} y(0) \\
& \quad+\left.\sum_{j=k+1}^{m}(-1)^{j-k-1} \int_{-r}^{0} \frac{\partial^{j-k-1}}{\partial \theta^{j-k-1}}\left(P_{j}(\theta) G_{\theta}^{(j)}(\theta, s)\right)\right|_{\theta=-0} y(s) d s=0,
\end{aligned}
$$

for $1 \leq k \leq m-1$, and 


$$
\begin{aligned}
& \left.\sum_{j=k+1}^{m}(-1)^{j-k-1} \frac{\partial^{j-k-1}}{\partial \theta^{j-k-1}}\left(P_{j}(\theta) G_{\theta}^{(j)}(\theta, 0)\right)\right|_{\theta=-r} y(0) \\
& \quad+\left.\sum_{j=k+1}^{m}(-1)^{j-k-1} \int_{-r}^{0} \frac{\partial^{j-k-1}}{\partial \theta^{j-k-1}}\left(P_{j}(\theta) G_{\theta}^{(j)}(\theta, s)\right)\right|_{\theta=-r} y(s) d s=0,
\end{aligned}
$$

for $0 \leq k \leq m-1$. The last conditions and the definition of the operator $L$ yield the equalities

$$
\begin{aligned}
& \sum_{j=0}^{m}(-1)^{j}\left(\frac{\partial^{j}}{\partial \theta^{j}}\left(P_{j}(\theta) G_{\theta}^{(j)}(\theta, 0)\right) y(0)+\frac{\partial^{j}}{\partial \theta^{j}} \int_{-r}^{0} P_{j}(\theta) G_{\theta}^{(j)}(\theta, s) y(s) d s\right) \\
&= y(\theta), \quad \theta \in[-r, 0) \\
& Q_{0}\left(G(0,0) y(0)+\int_{-r}^{0} G(0, s) y(s) d s\right) \\
& \quad+\sum_{j=1}^{m}(-1)^{j-1}\left(\frac{\partial^{j-1}}{\partial \theta^{j-1}}\left(P_{j}(\theta) G_{\theta}^{(j)}(\theta, 0)\right) y(0)\right. \\
&\left.+\frac{\partial^{j-1}}{\partial \theta^{j-1}} \int_{-r}^{0} P_{j}(\theta) G_{\theta}^{(j)}(\theta, s) y(s) d s\right)\left.\right|_{\theta=-0}=y(0),
\end{aligned}
$$

Under the conditions of smoothness of the matrix-function $G(\theta, s), \theta, s \in$ $[-r, 0)$, in the statement of Lemma 3 , equality (3.12) can be written in the form

$$
\begin{gathered}
\sum_{j=0}^{m}(-1)^{j} \frac{\partial^{j}}{\partial \theta^{j}}\left(P_{j}(\theta) G_{\theta}^{(j)}(\theta, 0)\right) y(0)+\sum_{j=0}^{m-1}(-1)^{j} \int_{-r}^{0} \frac{\partial^{j}}{\partial \theta^{j}}\left(P_{j}(\theta) G_{\theta}^{(j)}(\theta, s)\right) y(s) d s \\
+(-1)^{m} \frac{\partial}{\partial \theta} \int_{-r}^{0} \frac{\partial^{m-1}}{\partial \theta^{m}}\left(P_{m}(\theta) G_{\theta}^{(m)}(\theta, s)\right) y(s) d s=y(\theta), \quad \theta \in[-r, 0) .
\end{gathered}
$$

For the last integral we obtain

$$
\begin{aligned}
& \frac{\partial}{\partial \theta} \int_{-r}^{0} \frac{\partial^{m-1}}{\partial \theta^{m-1}}\left(P_{m}(\theta) G_{\theta}^{(m)}(\theta, s)\right) y(s) d s \\
& =\int_{-r}^{\theta} \frac{\partial^{m}}{\partial \theta^{m}}\left(P_{m}(\theta) G_{\theta}^{(m)}(\theta, s)\right) y(s) d s+\int_{\theta}^{0} \frac{\partial^{m}}{\partial \theta^{m}}\left(P_{m}(\theta) G_{\theta}^{(m)}(\theta, s)\right) y(s) d s \\
& \quad+\left(\left.\frac{\partial^{m-1}}{\partial \theta^{m-1}}\left(P_{m}(\theta) G_{\theta}^{(m)}(\theta, s)\right)\right|_{s=\theta-0}-\left.\frac{\partial^{m-1}}{\partial \theta^{m-1}}\left(P_{m}(\theta) G_{\theta}^{(m)}(\theta, s)\right)\right|_{s=\theta+0}\right) y(\theta), \\
& \quad \theta \in[-r, 0) .
\end{aligned}
$$


As a result, we have equality (3.12) in a new form

$$
\begin{aligned}
\sum_{j=0}^{m}(-1)^{j} \frac{\partial^{j}}{\partial \theta^{j}}\left(P_{j}(\theta) G_{\theta}^{(j)}(\theta, 0)\right) y(0)+\sum_{j=0}^{m-1}(-1)^{j} \int_{-r}^{0} \frac{\partial^{j}}{\partial \theta^{j}}\left(P_{j}(\theta) G_{\theta}^{(j)}(\theta, s)\right) y(s) d s \\
\quad+(-1)^{m}\left(\int_{-r}^{\theta} \frac{\partial^{m}}{\partial \theta^{m}}\left(P_{m}(\theta) G_{\theta}^{(m)}(\theta, s)\right) y(s) d s+\int_{\theta}^{0} \frac{\partial^{m}}{\partial \theta^{m}}\left(P_{m}(\theta) G_{\theta}^{(m)}(\theta, s)\right) y(s) d s\right. \\
\left.\quad+\left.\frac{\partial^{m-1}}{\partial \theta^{m-1}}\left(P_{m}(\theta) G_{\theta}^{(m)}(\theta, s)\right)\right|_{s=\theta-0} y(\theta)-\left.\frac{\partial^{m-1}}{\partial \theta^{m-1}}\left(P_{m}(\theta) G_{\theta}^{(m)}(\theta, s)\right)\right|_{s=\theta+0} y(\theta)\right) \\
=y(\theta), \quad \theta \in[-r, 0),
\end{aligned}
$$

Therefore we conclude

$$
\begin{aligned}
\sum_{j=0}^{m} & (-1)^{j} \frac{\partial^{j}}{\partial \theta^{j}}\left(P_{j}(\theta) G_{\theta}^{(j)}(\theta, 0)\right) y(0)+\sum_{j=0}^{m}(-1)^{j} \int_{-r}^{0} \frac{\partial^{j}}{\partial \theta^{j}}\left(P_{j}(\theta) G_{\theta}^{(j)}(\theta, s)\right) y(s) d s \\
& +(-1)^{m} \int_{\theta}^{0} \frac{\partial^{m}}{\partial \theta^{m}}\left(P_{m}(\theta) G_{\theta}^{(m)}(\theta, s)\right) y(s) d s \\
& +(-1)^{m}\left(\left.\frac{\partial^{m-1}}{\partial \theta^{m-1}}\left(P_{m}(\theta) G_{\theta}^{(m)}(\theta, s)\right)\right|_{\theta=s+0}\right. \\
& \left.-\left.\frac{\partial^{m-1}}{\partial \theta^{m-1}}\left(P_{m}(\theta) G_{\theta}^{(m)}(\theta, s)\right)\right|_{\theta=s-0}-(-1)^{m} I_{n}\right) y(\theta)=0, \quad \theta \in[-r, 0) .
\end{aligned}
$$

For the fulfillment of the last equality for any function $y \in H$, one needs that the matrix-function $G(\theta, s), \theta \in[-r, 0)$, for $\theta \neq s, s \in[-r, 0)$, satisfies the matrix differential equation (3.5), and the discontinuity of its derivative is determined by formula (3.4). Also the matrix-function $G(\theta, 0), \theta \in[-r, 0)$, satisfies the equation

$$
\sum_{j=0}^{m}(-1)^{j} \frac{d^{j}}{d \theta^{j}}\left(P_{j}(\theta) G_{\theta}^{(j)}(\theta, 0)\right)=0, \quad \theta \in[-r, 0) .
$$

The conditions (3.10), (3.11) give us that the matrix-function $G(\theta, s)$, $\theta \in[-r, 0), \quad \theta \neq s, \quad s \in[-r, 0)$, satisfies the boundary conditions (3.6), (3.7), and the matrix-function $G(\theta, 0), \theta \in[-r, 0)$, satisfies the boundary conditions

$$
\left.Q_{k} \frac{\partial^{k} G_{\theta}^{(k)}(\theta, 0)}{\partial \theta^{k}}\right|_{\theta=-0}+\left.\sum_{j=k+1}^{m}(-1)^{j-k-1} \frac{\partial^{j-k-1}}{\partial \theta^{j-k-1}}\left(P_{j}(\theta) G_{\theta}^{(j)}(\theta, 0)\right)\right|_{\theta=-0}=0
$$


for $1 \leq k \leq m-1$, and

$$
\left.\sum_{j=k+1}^{m}(-1)^{j-k-1} \frac{\partial^{j-k-1}}{\partial \theta^{j-k-1}}\left(P_{j}(\theta) G_{\theta}^{(j)}(\theta, 0)\right)\right|_{\theta=-r}=0, \quad 0 \leq k \leq m-1,
$$

When $m=1$, condition (3.15) is absent.

The condition (3.13) gives us the dependencies (3.8) and (3.9).

From the properties of the matrix-function $G(\theta, s), \theta \in[-r, 0), \theta \neq s$, $s \in[-r, 0)$, it follows that the matrix function $G(\theta, 0), \theta \in[-r, 0)$, defined by formula (3.8) satisfies equation (3.14), and the boundary conditions (3.15), and (3.16).

Theorem 1. Let the conditions $(\mathrm{H} 1)-(\mathrm{H} 3)$ be satisfied and let $\operatorname{Ker} U=\{0\}$. Then for a positive $\alpha$ the solution of the boundary value problem for the integrodifferential equation (2.2), (2.3) exists, is unique and continuously depends on $\varphi \in H$.

Proof. Since the operator $U^{*}$ is completely continuous and because of Lemma 3 it follows that the equation (3.1) is equivalent to the Fredholm equation of the second kind

$$
L^{-1} U^{*}(U x-\varphi)(\theta)+\alpha x(\theta)=0, \quad \theta \in[-r, 0] .
$$

If the homogeneous equation

$$
L^{-1} U^{*} U x+\alpha x=0,
$$

has a nontrivial solution, then one has $y=U x \neq 0$. Here $y$ is a solution of the equation

$$
U L^{-1} U^{*} y+\alpha y=0 .
$$

Since $U L^{-1} U^{*}$ is a self-adjoint positive operator, the eigenvalues are nonnegative. This is a contradiction.

Consequently, for $\alpha>0$ there exists a bounded operator $\left(L^{-1} U^{*} U+\alpha I\right)^{-1}$. Then the solution $x_{\alpha}$ of equation (1.6) exists, is unique, continuously depends on $\varphi \in H$, and is determined by the formula $x_{\alpha}=\left(L^{-1} U^{*} U+\alpha I\right)^{-1} L^{-1} U^{*} \varphi$, $\varphi \in H$. By taking into account a connection between equation (3.1) and the boundary value problem (2.2), (2.3), the proof of the theorem is complete.

For functional-differential equations conditions implying $\operatorname{Ker} U=\{0\}$ were investigated in [1].

From Theorem 1 it follows that the regularized solution of the above given ill-posed problem exits and is unique. It coincides with the solution of the boundary value problem (2.2)-(2.3) for the integro-differential equation. In the 
next paragraph it is shown that this boundary value problem is equivalent to the boundary value problem for a system of functional-differential equations.

\section{A boundary value problem for a system of functional-differential equations}

In the definition of the operator $U$ we use the Cauchy matrix $V$, which is defined implicitly by the functional-differential equation (1.1). When finding solutions of the ill-posed problem for the differential equation with one concentrated delay it is shown in the paper $[2,3]$ that one can replace equation (3.1) by an equivalent boundary value problem for ordinary differential equations, with coefficients that can be defined explicitly. It will be shown below that the boundary value problem for the system of functional-differential equations arises when finding solutions of the ill-posed problem for the functional-differential equation.

Lemma 4. Let the conditions $(\mathrm{H} 1)$ and $(\mathrm{H} 2)$ be satisfied. Then for the operator $U: W_{2}^{1}\left([-r, 0], \boldsymbol{R}^{n}\right) \rightarrow W_{2}^{1}\left([-r, 0], \boldsymbol{R}^{n}\right)$ the following representation holds

$$
U=T_{0}+T_{1} \circ T_{2}
$$

where the operators $T_{0}: W_{2}^{1}\left([-r, 0], \boldsymbol{R}^{n}\right) \rightarrow W_{2}^{1}\left([-r, 0], \boldsymbol{R}^{n}\right), \quad T_{1}: H \rightarrow$ $W_{2}^{1}\left([-r, 0], \boldsymbol{R}^{n}\right), T_{2}: W_{2}^{1}\left([-r, 0], \boldsymbol{R}^{n}\right) \rightarrow H$ are determined by the formulas:

$$
\begin{aligned}
& \left(T_{0} x\right)(\theta)=V(\theta,-r) x(0), \quad \theta \in[-r, 0], \\
& \left(T_{1} x\right)(\theta)=\int_{-r}^{\theta} V(\theta, s) x(s) d s, \quad \theta \in[-r, 0], \\
& \left(T_{2} x\right)(\theta)=\int_{\theta}^{-0}\left[d_{s} \eta(\theta, s-\theta-r)\right] x(s), \quad \theta \in[-r, 0), \\
& \left(T_{2} x\right)(0)=0,
\end{aligned}
$$

Proof. Let us transform the representation (1.4)

$$
\begin{aligned}
(U x)(\theta)= & V(\theta,-r) x(0)+\int_{-r}^{-0}\left[d_{s} \int_{-r}^{\theta} V(\theta, \tau) \eta(\tau, s-\tau-r) d \tau\right] x(s) \\
= & V(\theta,-r) x(0)+\int_{-r}^{\theta} V(\theta, \tau)\left(\int_{-r}^{-0}\left[d_{s} \eta(\tau, s-\tau-r)\right] x(s)\right) d \tau \\
= & V(\theta,-r) x(0)+\int_{-r}^{\theta} V(\theta, \tau)\left(\int_{\tau}^{-0}\left[d_{s} \eta(\tau, s-\tau-r)\right] x(s)\right) d \tau \\
& \theta \in[-r, 0], x \in W_{2}^{1}\left([-r, 0], \boldsymbol{R}^{n}\right) .
\end{aligned}
$$


The definitions of the operators $T_{0}, T_{1}$, and $T_{2}$ given in (4.2) imply the representations (4.1) hold.

Lemma 5. Let the conditions $(\mathrm{H} 1)$ and $(\mathrm{H} 2)$ be satisfied. Then for the operator $U^{*}: H \rightarrow H$ we have the following representation

$$
U^{*}=T_{0}^{*}+T_{2}^{*} \circ T_{1}^{*},
$$

where the operators $T_{0}^{*}: H \rightarrow H, \quad T_{1}^{*}: H \rightarrow H^{1}=W_{2}^{1}\left([-r, 0), \boldsymbol{R}^{n}\right) \times \boldsymbol{R}^{n}$, $T_{2}^{*}: H^{1} \rightarrow H$ are determined by the formulas:

$$
\begin{aligned}
& \left(T_{0}^{*} y\right)(\theta)=0, \quad \theta \in[-r, 0), \\
& \left(T_{0}^{*} y\right)(0)=V^{\top}(0,-r) y(0)+\int_{-r}^{0} V^{\top}(s,-r) y(s) d s, \\
& \left(T_{1}^{*} y\right)(\theta)=V^{\top}(0, \theta) y(0)+\int_{\theta}^{0} V^{\top}(s, \theta) y(s) d s, \quad \theta \in[-r, 0), \\
& \left(T_{1}^{*} y\right)(0)=0, \\
& \left(T_{2}^{*} y\right)(\theta)=\frac{d}{d \theta} \int_{-r}^{\theta}(\eta(s, \theta-s-r)-\eta(s,-r))^{\top} y(s) d s, \quad \theta \in[-r, 0), \\
& \left(T_{2}^{*} y\right)(0)=0 .
\end{aligned}
$$

Proof. The operator $T_{0}$ defined by the formula (4.2) admits an extension $T_{0}: H \rightarrow H$ defined by the same formula. For this extension the equality

$$
\begin{aligned}
\left(T_{0} x, y\right) & =y^{\top}(0) V(0,-r) x(0)+\int_{-r}^{0} y^{\top}(s) V(s,-r) d s x(0) \\
& =\left(x, T_{0}^{*} y\right), \quad x, y \in H,
\end{aligned}
$$

holds, from which we find a representation for the adjoint operator $T_{0}^{*}$. The representation for the adjoint operator $T_{1}^{*}$ follows from the equality

$$
\begin{aligned}
\left(T_{1} x, y\right) & =\int_{-r}^{0}\left(y^{\top}(0) V(0, \theta)+\int_{\theta}^{0} y^{\top}(s) V(s, \theta) d s\right) x(\theta) d \theta \\
& =\left(x, T_{1}^{*} y\right), \quad x, y \in H .
\end{aligned}
$$

The codomain of the operator $T_{1}^{*}$ belongs to the space $H^{1}=W_{2}^{1}\left([-r, 0), \boldsymbol{R}^{n}\right) \times$ $\boldsymbol{R}^{n}$. equality

When finding a representation for the adjoint operator $T_{2}^{*}$ from the

$$
\left(T_{2} x, y\right)=\left(x, T_{2}^{*} y\right), \quad x \in W_{2}^{1}\left([-r, 0], \boldsymbol{R}^{n}\right), y \in H^{1}
$$


at first we rewrite the formula

$$
\begin{aligned}
\left(T_{2} x, y\right)= & \int_{-r}^{0} y^{\top}(\theta)\left(\int_{\theta}^{-0}\left[d_{s} \eta(\theta, s-\theta-r)\right] x(s)\right) d \theta \\
= & \int_{-r}^{0} y^{\top}(\theta)(\eta(\theta,-0-\theta-r)-\eta(\theta,-r)) d \theta x(0) \\
& -\int_{-r}^{0} y^{\top}(\theta) \int_{\theta}^{-0}(\eta(\theta, s-\theta-r)-\eta(\theta,-r)) x^{\prime}(s) d s d \theta \\
= & \int_{-r}^{0} \int_{-r}^{s} y^{\top}(\theta)(\eta(\theta, s-\theta-r)-\eta(\theta,-r)) d \theta x^{\prime}(s) d s \\
= & \int_{-r}^{0} \frac{d}{d \theta} \int_{-r}^{\theta} y^{\top}(s)(\eta(s, \theta-s-r)-\eta(\theta,-r)) d s x(\theta) d \theta \\
= & \left(x, T_{2}^{*} y\right), \quad x, y \in H^{1} .
\end{aligned}
$$

This gives us the representation of the adjoint operator $T_{2}^{*}$. Finally, the use of representation (4.1) for the operator $U$ implies that formula (4.3) holds.

According to Theorem 1 we have that for an arbitrary function $\varphi \in H$ the equation (3.1) has a unique solution $x=x_{\alpha} \in W_{2}^{2 m}$ continuously depending on $\varphi$. This equation is equivalent to the system

$$
\begin{aligned}
& U^{*}(U x-\varphi)(\theta)+\alpha(L x)(\theta)=0, \quad \theta \in[-r, 0), \\
& U^{*}(U x-\varphi)(0)+\alpha(L x)(0)=0, \\
& F_{s}^{1}(x)=0, \quad 1 \leq s \leq m-1, \quad F_{s}^{2}(x)=0, \quad 0 \leq s \leq m-1 .
\end{aligned}
$$

By taking into account representation (4.3) for the operator $U^{*}$ and by introducing the function $\chi(\theta)=(U x)(\theta), \theta \in[-r, 0]$, the last system can be reduced to the form

$$
\begin{aligned}
& T_{2}^{*} T_{1}^{*}(\chi-\varphi)(\theta)+\alpha(L x)(\theta)=0, \quad \theta \in[-r, 0), \\
& T_{0}^{*}(\chi-\varphi)(0)+\alpha(L x)(0)=0, \\
& F_{s}^{1}(x)=0, \quad 1 \leq s \leq m-1, \quad F_{s}^{2}(x)=0, \quad 0 \leq s \leq m-1 .
\end{aligned}
$$

By introducing the functions

$$
\begin{aligned}
& \hat{\chi}(\theta)=\left(T_{1}^{*} \chi\right)(\theta), \quad \hat{z}(\theta)=\left(T_{1}^{*} \varphi\right)(\theta), \quad \theta \in[-r, 0), \\
& \hat{\chi}(0)=\left(T_{1}^{*} \chi\right)(-0), \quad \hat{z}(0)=\left(T_{1}^{*} \varphi\right)(-0),
\end{aligned}
$$

we have the last system of equations in the form 


$$
\begin{aligned}
& T_{2}^{*}(\hat{\chi}-\hat{z})(\theta)+\alpha(L x)(\theta)=0, \quad \theta \in[-r, 0), \\
& T_{0}^{*}(\chi-\varphi)(0)+\alpha(L x)(0)=0, \\
& F_{s}^{1}(x)=0, \quad 1 \leq s \leq m-1, \quad F_{s}^{2}(x)=0, \quad 0 \leq s \leq m-1 .
\end{aligned}
$$

We proceed to the proof of the Main Theorem, where the index $k$ is omitted in the statement and in the proof.

Proof of the Main Theorem. By taking into account the definitions of the operators $T_{2}^{*}$ and $L$, we rewrite equation (4.4) in the implicit form (1.6).

From the definition of the function $\hat{\chi}$ we have

$$
\hat{\chi}(\theta)=V^{\top}(0, \theta) \chi(0)+\int_{\theta}^{0} V^{\top}(\xi, \theta) \chi(\xi) d \xi, \quad \theta \in[-r, 0) .
$$

This equation is equivalent to the differential equation

$$
\frac{d \hat{\chi}(\theta)}{d \theta}=\frac{\partial V^{\top}(0, \theta)}{\partial \theta} \chi(0)-\chi(\theta)+\int_{\theta}^{0} \frac{\partial V^{\top}(\xi, \theta)}{\partial \theta} \chi(\xi) d \xi, \quad \theta \in[-r, 0),
$$

with the boundary condition

$$
\hat{\chi}(-0)=\chi(0) .
$$

The Cauchy matrix $V$ satisfies the integral equation [1]

$$
V(\xi, \theta)=I_{n}-\int_{\theta}^{\xi} V(\xi, s) \eta(s, \theta-s) d s, \quad-r \leq \theta \leq \xi<0 .
$$

The condition $\eta(\theta, 0)=0, \theta \in[-r, 0)$, gives us that

$$
\frac{\partial V^{\top}(\xi, \theta)}{\partial \theta}=\int_{\theta}^{\xi}\left[\left.d_{s} \eta^{\top}(\tau, \theta-s)\right|_{\tau=s}\right] V^{\top}(\xi, s), \quad-r \leq \theta \leq \xi<0 .
$$

Consequently, we have

$$
\begin{aligned}
& \frac{\partial V^{\top}(0, \theta)}{\partial \theta} \chi(0)+\int_{\theta}^{0} \frac{\partial V^{\top}(\xi, \theta)}{\partial \theta} \chi(\xi) d \xi \\
& \quad=\int_{\theta}^{0}\left[\left.d_{s} \eta^{\top}(\tau, \theta-s)\right|_{\tau=s}\right] V^{\top}(0, s) \chi(0)+\int_{\theta}^{0} \int_{\theta}^{\xi}\left[\left.d_{s} \eta^{\top}(\tau, \theta-s)\right|_{\tau=s}\right] V^{\top}(\xi, s) \chi(\xi) d \xi \\
& \quad=\int_{\theta}^{0}\left[\left.d_{s} \eta^{\top}(\tau, \theta-s)\right|_{\tau=s}\right]\left(\int_{s}^{0} V^{\top}(\xi, s) \chi(\xi) d \xi+V^{\top}(0, s) \chi(0)\right) \\
& \quad=\int_{\theta}^{0}\left[\left.d_{s} \eta^{\top}(\tau, \theta-s)\right|_{\tau=s}\right] \hat{\chi}(s), \quad \theta \in[-r, 0) .
\end{aligned}
$$

As a result, equation (4.7) takes the form (1.7). 
Similarly, from the definition of the function $\hat{z}$ we find that it satisfies the differential equation (1.8) with the boundary condition

$$
\hat{z}(-0)=\varphi(0) .
$$

The definition of the function $\chi$ leads to

$$
\chi(\theta)=V(\theta,-r) x(0)+\int_{-r}^{\theta} V(\theta, \xi) \hat{x}(\xi) d \xi, \quad \theta \in[-r, 0) .
$$

The previous equation is equivalent to the differential equation

$$
\frac{d \chi(\theta)}{d \theta}=\frac{\partial V(\theta,-r)}{\partial \theta} x(0)+\hat{x}(\theta)+\int_{-r}^{\theta} \frac{\partial V(\theta, \xi)}{d \theta} \hat{x}(\xi) d \xi, \quad \theta \in[-r, 0),
$$

with the boundary condition

$$
\chi(-r)=x(0) .
$$

The Cauchy matrix $V$ satisfies the differential equation [1]

$$
\begin{aligned}
\frac{\partial V(\theta, \xi)}{d \theta} & =\int_{-r}^{0}\left[d_{s} \eta(\theta, s)\right] V(\theta+s, \xi) \\
& =\int_{\xi-\theta}^{0}\left[d_{s} \eta(\theta, s)\right] V(\theta+s, \xi), \quad-r \leq \xi \leq \theta<0 .
\end{aligned}
$$

By taking into account these equations we rewrite the equation (4.8) in the form

$$
\begin{aligned}
\hat{x}(\theta) & +\int_{-r}^{0}\left[d_{s} \eta(\theta, s)\right] \int_{\xi-\theta}^{0} V(\theta+s, \xi) \hat{x}(\xi) d \xi \\
& =\frac{d \chi(\theta)}{d \theta}-\int_{-r-\theta}^{0}\left[d_{s} \eta(\theta, s)\right] V(\theta+s,-r) x(0), \quad \theta[-r, 0) .
\end{aligned}
$$

Let us reduce the previous equation to the form

$$
\begin{aligned}
\hat{x}(\theta) & +\int_{-r-\theta}^{0}\left[d_{s} \eta(\theta, s)\right]\left(\int_{-r}^{\theta+s} V(\theta+s, \xi) \hat{x}(\xi) d \xi+V(\theta+s,-r) x(0)\right) \\
& =\frac{d \chi(\theta)}{d \theta}, \quad \theta \in[-r, 0) .
\end{aligned}
$$

So equation (1.8) and the definition of the function $\hat{x}$ give us (1.9).

Let us find the implicit form of the boundary condition (4.5). We have

$$
\begin{aligned}
T_{0}^{*}(\chi-\varphi)(0) & =V^{\top}(0,-r)(\chi(0)-\varphi(0))+\int_{-r}^{0} V^{\top}(\xi,-r)(\chi(\xi)-\varphi(\xi)) d \xi \\
& =T_{1}^{*}(\chi-\varphi)(-r)=\hat{\chi}(-r)-\hat{z}(-r) .
\end{aligned}
$$


Consequently, the boundary condition (4.5) coincides with (1.10) and the boundary conditions (4.6) coincide with conditions (1.11), (1.12). The remaining boundary conditions appear by changing the integral equations to the differential equations.

The equivalence of the boundary value problem for integro-differential equation to the boundary value problem for a system of functional-differential equations and Theorem 1 leads to the existence and uniqueness of solution of the boundary value problem (1.6)-(1.12).

\section{Application}

The boundary value problem (1.6)-(1.12) for the functional-differential equation of the general form (1.1) has a complicated structure. This boundary value problem can be simplified for differential equations with multiple delays.

Example 1. Let us consider the nonautonomous differential equation with some constant delays

$$
\frac{d x(t)}{d t}=\sum_{j=0}^{p} A_{j}(t) x\left(t-\tau_{j}\right), \quad t \in(-\infty, 0],
$$

where $x:(-\infty, 0] \rightarrow \boldsymbol{R}^{n}, 0=\tau_{0}<\tau_{1}<\cdots<\tau_{p}=r$, det $A_{p}(t) \neq 0$ for $t \in(-\infty, 0]$, $p \in N$. The stabilizing functional is defined by the formula (1.5) and $m=1$.

The equation (5.1) is equivalent to (1.1) when

$$
\begin{aligned}
\eta(t, s)= & -\sum_{j=0}^{p-1} \mathbf{I}_{\left(-\tau_{p},-\tau_{j}\right)}(s) A_{j}(t), \quad \eta\left(t,-\tau_{p}\right)=-\sum_{j=0}^{p} A_{j}(t), \\
& -\tau_{p}<s<0, t \in(-\infty, 0],
\end{aligned}
$$

where $\mathbf{I}_{E}(\cdot)$ is an indicator of the set $E$.

Then the statement of the Main Theorem holds. The boundary value problem (1.6)-(1.12) is equivalent to the system of differential equation with deviating arguments

$$
\begin{aligned}
\alpha \frac{d}{d \theta}\left(P_{1}(\theta) \frac{d x_{k}(\theta)}{d \theta}\right)= & \alpha P_{0}(\theta) x_{k}(\theta) \\
& +\sum_{j=1}^{p} \mathbf{I}_{\left(-\tau_{j}, 0\right)}(\theta) A_{j k}^{\top}\left(\theta-\tau_{p}+\tau_{j}\right) \\
& \times\left(\hat{\chi}_{k}\left(\theta-\tau_{p}+\tau_{j}\right)-\hat{z}_{k}\left(\theta-\tau_{p}+\tau_{j}\right)\right),
\end{aligned}
$$




$$
\begin{aligned}
\frac{d \hat{\chi}_{k}(\theta)}{d \theta}= & -\sum_{j=0}^{p-1} \mathbf{I}_{\left(-\tau_{p},-\tau_{j}\right)}(\theta) A_{j k}^{\top}\left(\theta+\tau_{j}\right) \hat{\chi}_{k}\left(\theta+\tau_{j}\right)-\chi_{k}(\theta), \\
\frac{d \hat{z}_{k}(\theta)}{d \theta}= & -\sum_{j=0}^{p-1} \mathbf{I}_{\left(-\tau_{p},-\tau_{j}\right)}(\theta) A_{j k}^{\top}\left(\theta+\tau_{j}\right) \hat{z}_{k}\left(\theta+\tau_{j}\right)-x_{k+1}(\theta), \\
\frac{d \chi_{k}(\theta)}{d \theta}= & \sum_{j=0}^{p-1} \mathbf{I}_{\left(\tau_{j}-\tau_{p}, 0\right)}(\theta) A_{j k}(\theta) \chi_{k}\left(\theta-\tau_{j}\right) \\
& +\sum_{j=1}^{p} \mathbf{I}_{\left(-\tau_{p}, \tau_{j}-\tau_{p}\right)}(\theta) A_{j k}(\theta) x_{k}\left(\theta+\tau_{p}-\tau_{j}\right),
\end{aligned}
$$

with boundary conditions

$$
\begin{aligned}
& \alpha\left(Q_{0} x_{k}(0)+P_{1}(0) x_{k}^{\prime}(0)\right)+\hat{\chi}_{k}\left(-\tau_{p}\right)-\hat{z}_{k}\left(-\tau_{p}\right)=0, \quad x_{k}^{\prime}\left(-\tau_{p}\right)=0, \\
& \hat{\chi}_{k}(0)=\chi_{k}(0), \quad \hat{z}_{k}(0)=x_{k+1}(0), \quad \chi_{k}\left(-\tau_{p}\right)=x_{k}(0) .
\end{aligned}
$$

Here $x_{0}=\varphi \in H$ is a given function and $A_{j k}(\theta)=A_{j}\left((k+1) \tau_{p}+\theta\right), \theta \in\left[-\tau_{p}, 0\right]$, $0 \leq j \leq p, k \leq-1$.

The deviations of the arguments have different signs in the obtained system of differential equations. Numerical integration of these equations is a difficult problem, some approaches to its solution are considered in the works $[9,11]$. The presence of a small parameter at the highest derivative requires a special numerical method.

Example 2. Let us consider a problem of numerical finding regularized solutions of equation (5.1) for $p=2, \tau_{1}=\tau>0$, and $\tau_{2}=2 \tau$, on the finite interval $\left[-K \tau_{2}, 0\right], K \in N$, for a given value of the regularization parameter $\alpha$ and for a given initial function $\varphi \in H$. We assume that vrai $\sup _{t \in\left[-K \tau_{2}, 0\right]}\left|A_{2}(t)\right|<\infty$. For solving the boundary value problem (5.2), (5.3) we use the non-uniform mesh, the choice of which does not depend on $k,-K \leq k \leq-1$. The system of differential equations (5.2) is replaced by the following system of difference equations

$$
\begin{aligned}
x_{k}^{i+1}= & h_{i} P_{1}^{-1}\left(\vartheta_{i}\right) y_{k}^{i}+x_{k}^{i}, \\
y_{k}^{i+1}= & h_{i}\left(P_{0}\left(\vartheta_{i}\right) x_{k}^{i}+\alpha^{-1} H(i-N) A_{1 k}^{\top}\left(\vartheta_{i-N}\right)\left(\hat{\chi}_{k}^{i-N}-\hat{z}_{k}^{i-N}\right)\right. \\
& \left.+\alpha^{-1} A_{2 k}^{\top}\left(\vartheta_{i}\right)\left(\hat{\chi}_{k}^{i}-\hat{z}_{k}^{i}\right)\right)+y_{k}^{i}, \\
\hat{\chi}_{k}^{i+1}= & -h_{i}\left(A_{0 k}^{\top}\left(\vartheta_{i}\right) \hat{\chi}_{k}^{i}+H(N-i) A_{1 k}^{\top}\left(\vartheta_{i+N}\right) \hat{\chi}_{k}^{i+N}+\chi_{k}^{i}\right)+\hat{\chi}_{k}^{i},
\end{aligned}
$$




$$
\begin{aligned}
\hat{z}_{k}^{i+1}= & -h_{i}\left(A_{0 k}^{\top}\left(\vartheta_{i}\right) \hat{z}_{k}^{i}+H(N-i) A_{1 k}^{\top}\left(\vartheta_{i+N}\right) \hat{z}_{k}^{i+N}+x_{k+1}^{i}\right)+\hat{z}_{k}^{i}, \\
\chi_{k}^{i+1}= & h_{i}\left(A_{0 k}\left(\vartheta_{i}\right) \chi_{k}^{i}+H(i-N) A_{1 k}\left(\vartheta_{i}\right) \chi_{k}^{i-N}+H(N-i) A_{1 k}\left(\vartheta_{i}\right) x_{k}^{i+N}\right. \\
& \left.+A_{2 k}\left(\vartheta_{i}\right) x_{k}^{i}\right)+\chi_{k}^{i}, \quad i=0, \ldots, 2 N-1,
\end{aligned}
$$

where $H(\cdot)$ is the Heavyside step function, $h_{i}=\vartheta_{i+1}-\vartheta_{i}, 0 \leq i \leq 2 N-1$, and $\vartheta_{i}(0 \leq i \leq 2 N)$ is the mesh points of the interval $\left[-\tau_{2}, 0\right]$. The boundary conditions (5.3) take the form

$$
\begin{aligned}
& \alpha\left(Q_{0} x_{k}^{2 N}+P_{1}^{2 N} y_{k}^{2 N}\right)+\hat{\chi}_{k}^{0}-\hat{z}_{k}^{0}=0, \quad y_{k}^{0}=0, \\
& \hat{\chi}_{k}^{2 N}=\chi_{k}^{2 N}, \quad \hat{z}_{k}^{2 N}=x_{k+1}^{2 N}, \quad \chi_{k}^{0}=x_{k}^{2 N}, \quad-K \leq k \leq-1 .
\end{aligned}
$$

By using the methods of the work [12] we define the points of non-uniform mesh by the formulas

$$
\vartheta_{i}= \begin{cases}-2 \tau-\alpha^{1 / 2} \sigma_{1} \beta^{-1} \ln \left(1-\left(1-N^{-1}\right) 4 i / N\right), & i=0, \ldots, N / 4, \\ -\tau+\gamma_{1}+\rho(i-N / 4), & i=N / 4, \ldots, N / 2, \\ -\tau+\alpha^{1 / 2} \sigma_{2} \beta^{-1} \ln \left(1-\left(1-N^{-1}\right) 2(N-i) / N\right), & i=N / 2, \ldots, N\end{cases}
$$

and $\vartheta_{i}=\vartheta_{i-N}+\tau$ for $N+1 \leq i \leq 2 N$. Here $\gamma_{1}=-\tau+\alpha^{1 / 2} \sigma_{1} \beta^{-1} \ln N, \gamma_{2}=$ $-\alpha^{1 / 2} \sigma_{2} \beta^{-1} \ln N$, and $\rho=4\left(\gamma_{2}-\gamma_{1}\right) / N$, where $\beta, \sigma_{1}$ and $\sigma_{2}>0$ are some constants.

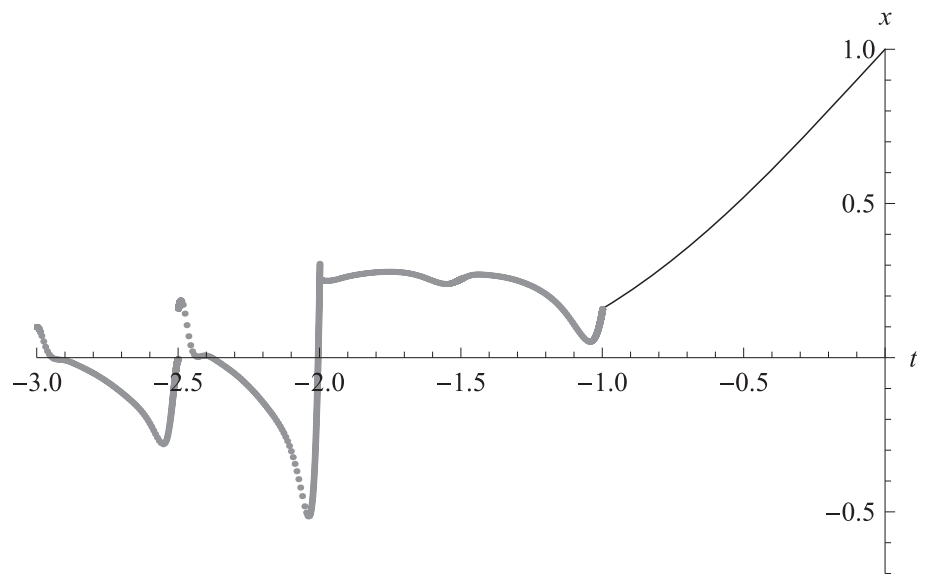

Fig. 1. Regularized solutions of equation (5.4) on $[-3,-1]$ for $\alpha=10^{-6}$ and $\varphi(\theta)=\sin (\theta)+1$, $\theta \in[-1,0]$. 
Let us consider the scalar equation

$$
x^{\prime}(t)=x(t)+t x(t-1 / 2)+\exp (-t) x(t-1)
$$

with a given initial function $\varphi(\theta)=\sin (\theta)+1, \theta \in[-1,0]$. The stabilizing functional is defined by the formulas $(1.5)$ for $m=1, Q_{0}=1, P_{1}(\theta)=1$ and $P_{0}(\theta)=3, \theta \in[-1,0]$. We will construct the regularized solution of equation (5.4) on the interval $[-3,-1]$.

The equation (5.4) coincides with (5.1) when $p=2, \quad \tau_{1}=0.5, \quad \tau_{2}=1$, $A_{0}(t)=1, A_{1}(t)=t$ and $A_{2}(t)=\exp (-t),-3 \leq t \leq 0$.

We choose the mesh parameters as follows $N=256, \beta=\exp (-5), \sigma_{1}=3 \beta$ and $\sigma_{2}=12 \beta$, and choose the value of the regularization parameter $\alpha=10^{-6}$. Then we find $\gamma_{1}=-0.983, \gamma_{2}=-0.0665, \rho=0.0143$.

The results of calculations are presented in fig. 1 .

\section{References}

[1] Hale, J. K. and Verduyn Lunel, S. M., Introduction to Functional-Differential Equations, Applied Mathematical Sciences, 99, Springer-Verlag, New York, 1993.

[2] Dolgii, Yu. F. and Putilova, E. N., Backward of Solutions of a Linear Differential Equation with Delay as an Ill-Posed Problem, Differentsial'nye Uravneniya, 29 (1993), 1317-1323.

[3] Dolgii, Yu. F. and Surkov, P. G., Asymptotics of Regularized Solutions of a Linear Nonautonomous System of Advanced Differential Equations, Differ. Equ., 46 (2010), 470-488.

[4] Tikhonov, A. N. and Arsenin, V. Ya., Solutions of ill-posed problems, Scripta Series in Mathematics, John Wiley \& Sons, New York-Toronto, Ont.-London, 1977.

[5] Vasin, V. V., Some tendencies in the Tikhonov regularization of ill-posed problems, J. Inverse Ill-Posed Probl., 14 (2006), 813-840.

[6] Dolgii, Yu. F., Characteristic Equation in the Problem of Asymptotic Stability of Periodic System with Aftereffect, Proc. Steklov Inst. Math. 2005, Dynamical Systems and Control Problems, suppl. 1, 82-94.

[ 7 ] Naimark, M. A., Linear differential operators, Part I: Elementary theory of linear differential operators, Frederick Ungar Publishing Co., New York, 1967.

[8] Yosida, K., Functional Analysis, Grundlehren der Mathematischen Wissenschaften, 123, Springer-Verlag, Berlin-New York, 1980.

[9] Azbelev, N., Maksimov, V. and Rakhmatullina, L., Introduction to the theory of linear functional differential equations, Advanced Series in Mathematical Science and Engineering, 3, World Federation Publishers Company, Atlanta, GA, 1995.

[10] Maksimov, V. P., Rumyantsev, A. N. and Shishkin, V. A., On constructing solutions of functional-differential systems with a guaranteed precision, Funct. Differ. Equ., 3 (1995), 135-144.

[11] Neverova, D. A. and Skubachevskii, A. L., On the Classical and Generalized Solutions of Boundary-Value Problems for Difference-Differential Equations with Variable Coeffcients, Math. Notes, 94 (2013), 653-667. 
[12] Miller, J. J., O'Riordan, E. and Shishkin, G. I., Fitted numerical methods for singular perturbation problems. Error estimates in the maximum error for linear problems in one and two dimensions, World Scientific Publishing Co., Inc., River Edge, NJ, 1996.

nuna adreso:

Yu. F. Dolgii

Institute of Mathematics and Computer Science

Ural Federal University

pr. Lenina 51, Yekaterinburg 620000

Russia

E-mail: Yurii.Dolgii@usu.ru

P. G. Surkov

Institute of Mathematics and Mechanics

Ural Branch of the Russian Academy of

Sciences

ul. S. Kovalevskoi 16, Yekaterinburg 620990

Russia

E-mail: Platon.Surkov@gmail.com

(Ricevita la 22-an de aprilo, 2013)

(Reviziita la 12-an de marto, 2014) 